\title{
Why Psychiatrist Leadership Should Understand and Engage Modern Media
}

\author{
Steve Schlozman ${ }^{1}$ (D) - Matthew Carron ${ }^{1} \cdot$ Eugene V. Beresin ${ }^{1}$
}

Published online: 25 April 2019

(C) Academic Psychiatry 2019

The current impressive and always-growing reach of modern media suggests that psychiatrists will necessarily be part of media coverage. To be clear, the authors of this article are very much in favor of mental health clinicians maintaining connection with and becoming even more skilled in the art of media literacy and media outreach, but even in the absence of this opinion, there is not really much of a choice. Media presence is everywhere. With this caveat in mind, this article will summarize how best to engage the new(ish) world of nearconstant media presence.

Entire college courses, indeed entire textbooks, are devoted to making sense of modern media. How to define media and how to engage media are two very different subjects. This article will attempt a cursory answer to both of these questions. Of course, much more can be said on these topics. To that end, this essay is also intended to pique the curiosity of readers to further explore this fascinating and, in many ways, as-yet unchartered frontier.

This article begins by attempting to define the term "media" in the modern context. This will be followed by a discussion of the presence of media training in medicine and especially psychiatry training, and then to an examination of the effects of these trainings on psychiatric residency program graduates. It is the contention of the authors that psychiatrists in leadership roles can and should actively contribute towards translating psychiatric training practices into higher-quality and more prolific media interactions. Finally, this article offers recommended guidelines for enhanced media engagement.

Steve Schlozman

SSchlozman@mgh.harvard.edu

1 Massachusetts General Hospital, Harvard Medical School, Boston, MA, USA

\section{How to Define "the Media"}

Obviously, this article would have been easier to write as recently as 20 years ago. The mainstream use of the Internet was in its infancy. Many of the readers of this article consumed their news and popular culture through print and on the television or the movie screen. If an issue with mental health implications found its way to the popular press, those who were asked to comment would have time to prepare statements, ample opportunities to complete the necessary research, and it was rare to experience the frenzied request for comments that has come to characterize the current experience of media engagement. Indeed, defining media before the end of the twentieth century would almost certainly limit any relevant discussion to newspapers, radio, television, and movies.

The irony of this realization is that the answer would likely be the same if one was asked ourselves to define media 40 years ago, or even 60 years ago. Many current trainees look at their slightly older attendings with wonder when those (barely) graying supervisors recall the first e-mail they received or the first website they visited. To these young doctors, this computer and especially Web-based technology has always been here. Historians have, in fact, been stymied to find comparisons. Some have asserted that the social changes that accompany the rapid changes in technology over the last 20 years are rivaled only by the introduction of the automobile. And still, the automobile was built and available to only a select few for a very long time. The new media become the main media in less than two decades.

For the purposes of this article, the definition of media will attempt to capture the wide array of information that is presented to the modern consumer of media outreach. In order to accomplish this goal, one can turn to the derivation of the word media itself. Media has its roots in the Latin term "Media," which translates to the "middle sheath" or "middle layer." In other words, the media is the intermediary-the 
middle part - of the means by which we communicate with our culture and by which our culture communicates with us.

Using this etymology as a guide, media includes print fiction and non-fiction including books, newspapers, magazines, and e-zines (magazines published entirely on the Internet). Media also includes the array of written informational and opinion pieces that populate Internet blogs, news sites, and magazine websites. It includes communication through social media apps such as Twitter, Facebook, and Instagram, providing immediate access to the public domain. Media further involves informational news television and/or streaming programs, entertainment-oriented news such as The John Oliver Show (also available both online and at pre-arranged viewing times), programs both online and on the major networks that convey information in fictional settings (In Treatment, for example), and the wide array of interactive media including online games, console-based video games, virtual reality sensory experiences, and most recently, interactive streaming television such as the recent Black Mirror episode Bandersnatch.

Summarizing the ways mental health professionals interact with all of these forms of media is nearly impossible and also somewhat moot, given the rapidity with which media changes. Any conclusions one reaches will have changed by the time of publication due to the pace of media itself. Because of these limitations, our discussion will attempt to apply to as many forms of media as possible but will focus primarily on traditional media, such as news outlets, and non-traditional formats, such as blogs and tweets. Still, one must keep in mind that new kinds of media that have yet to be imagined will, by definition, require frequent reevaluation of these guidelines. For now, however, it is useful to ask to what extent residency training programs currently offer formal didactics in media literacy and media engagement.

\section{What Do We Teach Physician Trainees About the Media?}

Most psychiatric educators value knowledge in how to interact with the media. However, studies have shown that while virtually all training programs in psychiatry acknowledge the importance of these skills, only about half of the psychiatric residencies in the USA have formal didactics focusing on these skills [1-3]. Furthermore, there is not always agreement as to what these skills entail, and there are no agreed upon curricula for teaching psychiatric trainees about media interaction. There is even debate as to the proper definition of the phrase, "media literacy" itself. A 2018 Academic Psychiatry article suggested that one can define media literacy as "the ability to access, analyze, evaluate and create media in a variety of forms" [3]. Given the appropriate inclusiveness of this definition, it is no surprise that the goals of pedagogic endeavors towards media literacy vary.

Historically, pediatricians have often found themselves in the middle of media coverage. Guidelines regarding national childhood educational efforts, proper child-rearing techniques, changes in nutritional recommendations, and the mental health needs of children have frequently called for pediatricians to promote and to offer opinions regarding these issues. Nevertheless, pediatric residencies find themselves facing similar challenges regarding media literacy despite their more reliably present media training during residency. A 2013 study suggested that while the majority of pediatricians acknowledged some formal media training during residency, only $38 \%$ found described their training as "good/excellent" [4]. In other words, even training programs that have long emphasized the roles of their graduates with regard to media interaction have not, at least according to the trainees themselves, consistently achieved their goals.

All of this speaks to the complex and difficult tasks that are inherent to any attempt at satisfactory media training for physicians. Physicians must, after all, maintain some degree of demarcation between their personal views and the views that are espoused in the office. And yet, the current frenzy of media attention to virtually every issue sometimes conflates with great fanfare health-related views and political views. Issues regarding firearms, corporeal punishment, LGBTQ rights, and microaggressions are all examples of stories where political agendas and health-related agendas coincide. Importantly, these are all issues where psychiatry is particularly called upon to inform the public, and yet many psychiatrists are not entirely comfortable commenting in the public sphere that media affords. To be sure, psychiatrists can always pass on the opportunity to be part of a psychiatry-related news story, but this reticence needs to be weighed against the potential harm that comes from not being an active part of the discussion and therefore potentially allowing rapid and widespread misinformation to go unchecked. This is not to say that one ought to always engage with media. Instead, one should be sure to at least consider the possibility of engaging. In this way, psychiatrists can be mindful of the role they play and the voice they bring to the discussion. The American Psychiatric Association in its Code of Ethics (Principles of Medical Ethics with Annotations Especially Applicable to Psychiatry Section 7.3) indicates that psychiatrists should not render psychiatric diagnoses to individuals they have not examined (The Goldwater Rule), yet Section 7.1 promotes the use of psychiatric expertise to advise governmental agencies and the public about "psychiatric issues that affect mental health and illness." And Section 7.2 encourages psychiatrists to engage in public education and use their professional expertise to do so [5]. So, in fact, one can 
argue that psychiatry as a medical discipline has a professional and ethical obligation to provide sound public health education, and to do so, psychiatrists need to have facility in successfully engaging with media outreach.

But how does one go about doing this? Is there a standard of care for media engagement? Is there a standard at all? Given the paucity of existing media training in psychiatric residencies, coupled with the variety among those programs that do, in fact, offer formal media literacy didactics, it seems clear that psychiatric leadership must embrace the importance of training all psychiatrists to be at the least competent when engaging in the public discourse that the media makes possible.

\section{What Is the Role of Psychiatric Leadership in Outreach to Traditional Media?}

Defining what exactly constitutes media, and even attempting to limit the discussion to so-called "traditional media," is increasingly problematic. News stories that are first reported in major publications are virtually always commented on through social media. On the other hand, comments on social media frequently create potent news stories in major publications. The recent events in Washington involving high school students, Native Americans, and the Black Hebrew Israelites are a perfect example of news and social media together creating a potentially inflammatory story. To this end, psychiatrists who interact with the media must recognize that any attempt they make to tease apart contact with traditional media - the major news publications, for example - and social media commentary, is, to some extent, not possible. On the other hand, it is the contention of the authors of this essay that psychiatrists are among those who are uniquely qualified to sort out the complexities of human actions, motivations, and the resulting impact.

In order to accomplish the goals of this essay, three cases are presented below. In each case, the authors include their best recollection of discussions with various media outlets. Importantly, these cases are meant to showcase the benefits as well as pitfalls inherent to media engagement by psychiatrists in leadership positions. As the reader will discover, these events also cross the boundaries of traditional and nontraditional media regardless of the original source.

\section{Case 1}

A psychiatrist was asked to offer guidance for children and teachers to discuss the fictional suicide that was viewed my millions on Netflix's immensely popular mini-series 13 Reasons Why. Because the series describes the reasons a teen girl takes her own life, there was concern and indeed evidence that the show could create suicidal ideation and death by suicide among the general public.
The psychiatrist was asked to comment during a phone interview. He accurately outlined for the reporter the need that parents and teachers openly discuss these issues with their teens and students. He also recommended that ample counseling be made available for at least a few weeks following viewing the program, and that great care should be taken to avoid romanticizing the suicide featured in the mini-series.

"But doctor," the interviewer interrupted, "You're saying that the contagion effect typically is time limited. Is that the case for this show, where it can be watched over and over?"

This is a perfect example of interpreting new forms of media through the lens of old media practices. Although it would have been optimal for the psychiatrist to have more actively considered the novelty of a streaming program, the messaging around the contagion effect, especially in the setting of older forms of screen-based entertainment, was the talking point that the psychiatrist had intended to emphasize. He had not been prepared to discuss the ways that a streaming program might change the recommendations regarding the well-known risks inherent with dramatized suicides. Therefore, while some of his talking points were indeed mentioned in the subsequent article [6], it was the streaming and therefore always-present nature of the program that the psychiatrist failed to consider. To his knowledge, no one had studied the effects of streaming entertainment and the suicide contagion. As a leader in psychiatry, the psychiatrist was able to note this absence of data to colleagues and professional organizations, suggest that studies be undertaken to answer the pertinent questions with regard to streaming media content, and then to take part in drafting recommendations for how to present 13 Reasons Why in a more public health minded fashion [7-9]. Because this article is focused on the particular need that leaders in psychiatry have to engage traditional media, it is important to note that had this clinician not been in a position of authority, he might not have been able to suggest and to take part in the necessary professional collaborations to better discuss public response to the possibility of a suicide contagion in a streaming setting.

\section{Case 2}

A psychiatrist was asked to comment on the mental health of the President-Elect of the USA. He was clear in his answer that the professional standards of psychiatry, as defined by the American Psychiatric Association, prohibited offering diagnostic opinions regarding public figures. He was then informed by the reporter that a graduate of the training program in which he had taught had recently led a national effort to declare the President-Elect unfit for duty [10], and he was asked whether he felt this was appropriate. Upon speaking with the graduate, he found the issue couched in terms of public health. A case could be made, his graduate explained, that if the President-Elect seemed unfit due to the trained eye 
of a competent expert in mental health, the health of the nation and, therefore, that of his patients were potentially at risk. As a result, the psychiatrist responded to a subsequent reporter neither agreeing nor disagreeing with the actions of the graduated resident, but instead noted the nuanced role that politics can play in the medical encounter [11].

As both of these cases illustrate, the leadership position of the psychiatrist made possible certain key changes that were necessary to approach the given events. These issues were then integrated into the overall training and practices of the department. Additionally, the psychiatrist was able to say with confidence that more thought was required for each issue and, at the same time, to acknowledge the newness of the issue when compared to similar issues from 20 years or 30 years ago.

\section{What Is the Role of Psychiatric Leadership when Engaging Non-traditional Media?}

For the purposes of this article, non-traditional media includes social media posts, the expanding world of online written and video blogs, and the audio formats of podcasts. It should be noted that these kinds of media are not firmly divorced from traditional media. Many well-known media outlets, for example, utilize expert bloggers, but the bloggers themselves are not subjected to the same or, at times, even any fact checking or editing. To this end, a psychiatrist might find his or her institution in the mast head of an online publication that has not been vetted either by editors at the magazine or the public relations department of the institution where he or she works.

Psychiatrists can offer unique and extremely helpful perspectives regarding important news events, but at the same time, psychiatrists must recognize that it is potentially reckless to put forth these perspectives without forethought as to the effects of these views when they are read, seen, or heard by the general public. As parents teach their children every day, anything that one puts online, even with the assumption that only certain people will even be able to access what is written or said, can be copied, pasted, and disseminated. It is therefore a good rule of thumb to actively consider these possibilities. One should explicitly state caveats that the views being expressed are those of the writer alone unless the writer is given permission to speak for many. Older journalistic practices did not dictate this necessity - it was often taken for granted that the views were the views of the writer. Blogs, however, perhaps because of the pace at which they can spread and the ways they can be changed without the author's knowledge, require greater caution. The following example illustrates these challenges.

\section{Case 3}

A psychiatrist wrote a blog that was critical of Oregon's proposed law that would allow psychologists to prescribe psychiatric medications [12]. The content of the blog was, by definition, controversial. The availability of Oregon-based psychologists as prescribers could measurably alleviate the pressure where a dearth of psychiatrists limited access by patients to potentially beneficial treatment. Furthermore, the psychiatrist who wrote the blog that was critical of the legislation did not reside in Oregon. He was therefore perceived as an outsider with little at stake other than professional turf in the discussion. Some of the comments were particularly vitriolic. One, in particular, came from a psychiatrist who used the term "fear mongering" in reference to the concerns expressed in the blog. As a result, local press in Oregon picked up the story and published excerpts from the blog as well as the critical comments. At this point, what had been local news in the Pacific Northwest became national news. After consultation with colleagues, the psychiatrist who had written the blog offered in the comment section of his original essay a civil but firm reminder that he was expressing his personal opinion and that others were free to disagree, but that his intent had never been to promulgate fear. The psychiatrist who had been critical of the blog post then utilized the same comment section to apologize and acknowledge a difference in opinions.

This example demonstrates the extent to which we must, in all circumstances, expect our engagement in non-traditional media to become entangled with traditional media. Psychiatrists in leadership roles need to be especially mindful of this possibility, given the greater visibility that leadership affords. We do have, in fact, some literature addressing these issues. Psychiatrists who communicate via platforms such as Twitter can bring their expertise to large audiences, thus promoting lifelong learning, patient care and advocacy, research data and collection, and greater impact of scholarly data [13]. However, as the case above makes clear, the opportunity to have one's words and intentions misconstrued, deliberately or otherwise, is exponentially greater in the online community. Articles and opinion pieces in more formal academic and professional settings do not share this same vulnerability.

\section{What Now?}

What constitutes media is constantly changing, even as the physician's roles remain relatively consistent. Physicians treat patients in order to minimize suffering and maximize wellbeing. Physicians render opinions towards preserving the health of the community. For highly visible physicians in positions of leadership, the opportunity to publicize these opinions and educate the public is especially potent. Additionally, the extent to which psychiatry and culture intermingle 
suggests that psychiatrists might very well find themselves in the midst of the new media trends. Is it possible, given the rapid pace of change, to construct nimble guidelines for media engagement?

The answer to this question is that it is both possible and absolutely necessary. The omni-present reach and the rapid dissemination of modern media suggest that media literacy is now a matter of public health. Proper engagement of the media is in keeping with the goals of the modern physician. Patients and communities have access to physician's opinions and directives as never before. Physicians should recognize this powerful tool and make use of it. In doing this, the following guidelines are recommended for psychiatrists and other physicians who wish to engage more actively in media efforts:

- Physicians should review with their departments how to respond to media interview requests for TV, radio, or online pieces; how to write blogs or op eds; and how to use social media productively.

- Physicians should understand how to craft educational material for the public.

- Physicians need to be wary of how their comments can be interpreted and misinterpreted and the subsequent implications of these interpretations for the professional reputation of the physician, the department, and the affiliated medical school.

- Physicians should make every effort to discuss with colleagues what they are considering saying or writing in the public sphere and prioritize their goals and objectives to further the advocacy missions they wish to pursue.

- All physicians who interact with the media need to follow up on their own media engagement. If one writes or comments on a given issue, one needs to be aware of whether, to what extent, and in what light these thoughts are being digested. In other words, what is the impact? This may be as simple as examining the number of views or eliciting responses from colleagues and friends and contacts in the general public.

- All physicians who interact with the media need to beware of "trolls." A number of individuals will respond with highly inflammatory comments. One should expect that there will be people who will attack almost any ideological position. Think carefully about how to respond or if it is worth responding at all.

- Consult often with public relations. This may be the public affairs office attached to a hospital or medical school or through consultation with a private public relations firm. The entire professional field of health communications is based on formal training to assess these issues. Physicians need to be more willing to take advantage of and to learn from this expertise. Experts in public relations know how one should frame important points and, in particular, what wording should be for different media, what media outlets are best suited to express specific issues, and how physicians can pitch their message to an outlet in the most effective manner.

- All physicians who interact with the media need to should know when to disengage. If something one says or does has been co-opted in ways that are not consistent with the intent of the original ideas, one need to discuss options with colleagues, to assess whether one ought to continue the public discourse, or to simply stop the discussion.

- All physicians must actively remain abreast of new changes in the ways media is transmitted and assimilated.

There is tremendous opportunity in media engagement. There is also tremendous peril. This is perhaps especially the case for psychiatrists, given the potentially controversial nature of mental health topics. Those who hold leadership roles in psychiatry are more powerful and, therefore, also more vulnerable due to their greater visibility. Media holds great promise, but media engagement should be taken seriously and extremely thoughtfully. Psychiatric educators need to train their residents to feel comfortable and competent with media engagement, and in many cases, it will, in fact, be the residents themselves who will make the older generation aware of new forms of media. As with every new development in medicine, it will take the entire medical village to make sure this is done correctly. This kind of teamwork is nothing new. Physicians need to keep these guidelines in mind the next time they post a tweet, compose a blog, or accept an invitation to comment on air.

The world is listening now more than ever before.

\section{Compliance with ethical standards}

Disclosure On behalf of all authors, the corresponding author states that there is no conflict of interest.

\section{References}

1. Kutner L, Beresin EV. Reaching out: mass media techniques for child and adolescent psychiatrists. J Am Acad Child Adolesc Psychiatry. 2000;39(11):1452-4.

2. Olson C.K., Kutner L.A. \& Beresin E.V: Mass media outreach for child psychiatrists. In Beresin E.V. \& Olson, C.K. (Eds.), Child and adolescent psychiatry and the media. St. Louis, Missouri: Elsevier. 2018. 117-124.

3. Morris NP, Johansen SL, May M, Gold JA. Media-related education in psychiatry residency programs. Acad Psychiatry. 2018;42: 679-85.

4. Christakis DA, Frintner MP, Mulligan DA, Fuld GL, Olson LM. Media education in pediatric residencies: a national survey. Academic Pediatrics. 2013;13:55-8.

5. American Psychiatric Association: The principles of medical ethics with annotations especially applicable to psychiatry, Edition. 2013 
6. Todd C. https://www.self.com/story/13-reasons-why-season-twomental-health-experts-commentary. Accessed March 28, 2019

7. Schlozman S and Beresin G. https://www.mghclaycenter.org/ parenting-concerns/when-teens-talk-of-suicide-2/. Accessed March 28, 2019

8. Beresin G. https://www.mghclaycenter.org/hot-topics/7142/. Accessed March 28, 2019

9. American Academy of Child and Adolescent Psychiatry. https:// www.aacap.org/AACAP/Latest_News/2014/In-Response-to-13Reasons-Why.aspx. Accessed March 28, 2019

10. Ganeva T. https://www.salon.com/2018/12/04/trump-fans-havetrait-toddlers-grow-out-of-doctor-says_partner/. Accessed March 28, 2019
11. Martin K. https://www.medscape.com/viewarticle/907680_1. Accessed March 28, 2019

12. Schlozman S. https://www.psychologytoday.com/us/blog/grandrounds/201004/real-anxieties-about-psychologists-prescribingaint-about-turf. Accessed March 28, 2019

13. Choo EK, Ranney ML, Chan TM, Trueger NS, Walsh AE, Tegtmeyer K, et al. (2015). Twitter as a tool for communication and knowledge exchange in academic medicine: a guide for skeptics and novices. Medical Teacher. 2015;37(5):411-6.

Publisher's Note Springer Nature remains neutral with regard to jurisdictional claims in published maps and institutional affiliations. 